\title{
On the Monotonicity of the Speed of Random Walks on a Percolation Cluster of Trees*
}

\author{
Dayue CHEN Fuxi ZHANG
}

\begin{abstract}
We consider the simple random walk on the infinite cluster of the Bernoulli bond percolation of trees, and investigate the relation between the speed of the simple random walk and the retaining probability $p$ by studying three classes of trees. A sufficient condition is established for Galton-Watson trees.
\end{abstract}

\section{Introduction}

The simple random walk $\left\{X_{n}\right\}$ on graph $\mathcal{G}=(V, E)$ is defined as a Markov chain on the set $V$ of vertices with transition probability $p(x, y)=1 / d_{x}$ if $x, y \in V$ and there is an edge between them. The degree $d_{x}$ of vertex $x$ is the number of edges connecting $x$ to other vertices.

Let $o$ be a fixed vertex and $|x|$ the graphic distance between $o$ and $x$, i.e., the minimum number of edges in a path from $o$ to $x$. Suppose the simple random walk starts from $o$, i.e., $X_{0}=o$. We call $\lim _{n}\left|X_{n}\right| / n$, if it exists, the speed of the simple random walk. For the simple random walk in $Z^{d}$, the speed is zero. On the other hand the speed is $(d-1) /(d+1)$ for the simple random walk on the regular tree $T_{d}$. By regular tree $T_{d}$, we mean a tree that the degree of every vertex except the root is $d+1$. The degree of the root $o$ is $d$.

A non-trivial example is the simple random walk on a Galton-Watson tree. Let $\left\{p_{n}, n=0,1,2, \cdots\right\}$ be the offspring distribution of a Galton-Watson process. Each

\footnotetext{
${ }^{*}$ Supported in part by Grant G1999075106 from the Ministry of Science and Technology of
} China. 
individual produces offsprings independently according to the same law. There is a rooted tree for each realization, and the correspondence induces a probability measure in the set of rooted trees. A rooted tree, drawn according to this measure, is called a Galton-Watson tree. It is shown in [5] that the speed on a Galton-Watson tree is a constant a.s. More remarkably, an explicit formula is given when $p_{0}=0$.

$$
\text { Speed }=\sum_{k=1}^{\infty} p_{k} \frac{k-1}{k+1} .
$$

In general, let $q$ be the extinction probability, i.e., $q=\sum_{k} p_{k} q^{k}$, then

$$
\text { Speed }=\sum_{k=0}^{\infty} p_{k} \frac{k-1}{k+1} \frac{1-q^{k+1}}{1-q^{2}} .
$$

In $p$-Bernoulli bond percolation in $\mathcal{G}$, each edge of $\mathcal{G}$ is independently declared open with probability $p$ and closed with probability $1-p$. Thus a bond percolation $\omega$ is a random subset of $E$. We usually identify the percolation $\omega$ with the subgraph of $\mathcal{G}$ consisting of all open edges and their end-vertices. A connected component of this subgraph is called an open cluster, or simply a cluster. Let $p_{c}=p_{c}(\mathcal{G})=\inf \{p$ : there is an infinite cluster a.s.\}. By coupling, the infinite cluster is increasing in $p$. The probability that there is an infinite cluster is monotone in $p$. When $p \in\left(p_{c}, 1\right)$, with positive probability the open cluster $\mathcal{C}$ that contains $o$ is infinite.

The simple random walk on the infinite cluster of $Z^{d}$ is first studied in [3]. A more systematic investigation is initiated in [1]. We like to investigate the relation between the speed of the simple random walk on an infinite cluster and the retaining probability $p$ by observing three examples: the regular tree, Galton-Watson trees, and the binary tree with pipes, which is obtained by adding a pipe to each vertex of the binary tree. See Figure 1.

Proposition 1 The speed of the simple random walk on an infinite cluster of a regular tree is increasing in $p$.

The main idea of the proof is to use formula (1) and the decomposition of an infinite Galton-Watson tree as a backbone and bushes. As $p$ increases, the backbone gets larger, the mean size and the number of bushes get smaller. It is tempting to think that the monotone relation holds for a large class of graphs. 
Question A: Is the speed of the simple random walk on an infinite cluster of a transitive graph increasing in $p$ ?

We believe the answer is "yes". However, this statement can not be pushed any further, as we see in the following example of the binary tree with pipes. The binary tree is chosen because the extinction probability can be explicitly calculated. Assume that $p>1 / 2$ and the cluster containing the root $o$ is infinite. In addition to the backbone and bushes, there are also pipes of random length. The distribution of the length is geometric and the mean length of a pipe is $p /(1-p)$. Therefore, the larger the $p$ is, the longer a pipe is, and the longer the time for the excursion on a pipe is. As $p$ increases, there is a competition between the increase of time spent on the pipe and the decrease of time spent on the bush.

Example 2 The speed of the simple random walk on an infinite cluster of the binary tree with pipes is

$$
\frac{1}{3} \frac{(2 p-1)^{2}}{p^{2}+(1-p)^{2}} \quad \frac{1-p}{\left(2 p^{3}-6 p^{2}+3 p+3\right)}
$$

which is not monotone in $p$.

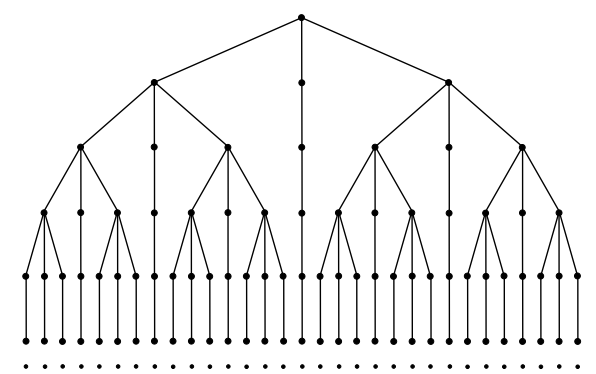

Figure 1: The binary with pipes

Very often a random environment (or graph) exhibits nice characters of a homogeneous environment (or a transitive graph). So we take the Galton-Watson trees. Let $\left\{p_{k}\right\}$ be the offspring distribution, and $m_{1}=\sum_{k} k p_{k}$ the mean number of offsprings. we always assume that $m_{1}>1$. Suppose the retaining probability $p>1 / m_{1}$ and the open cluster $\mathcal{C}_{o}$ of $p$-Bernoulli bond percolation containing the root is infinite. Run the simple random walk starting from the root. 
Theorem 3 Let $f(s)=\sum_{k} p_{k} s^{k}$ be the generating function and $f^{\prime}(s)$ the derivative of $f$. Suppose that

$$
\frac{(1-s) f^{\prime}(s)}{1-f(s)} \text { is increasing in } s
$$

for $s \in\left(1 / m_{1}, 1\right)$. Then the speed of the simple random walk on an infinite cluster of a Galton-Watson tree is an increasing function of $p$. Furthermore it is continuous for $p \in\left[1 / m_{1}, 1\right]$ and differentiable for $p \in\left(1 / m_{1}, 1\right)$.

It is not difficult to verify (2) in general, as we now examine some special cases.

Example 4 The geometric distribution, i.e. $p_{k}=a^{k}(1-a)$ for $0<a<1$. Then

$$
f(s)=\frac{1-a}{1-a s}, \quad \text { and } \quad \frac{(1-s) f^{\prime}}{1-f}=\frac{1-a}{1-a s}
$$

which is clearly increasing in $s$. With a little extra work one can show that the negative binomial distribution also satisfies (2).

Example 5 The Poisson distribution with parameter $\mu$. In this case, $f(s)=e^{s \mu-\mu}$ and $f^{\prime}(s)=\mu f(s)$. Then the derivative of $(1-s) f^{\prime} /(1-f)$ is $\mu \phi(s) f /(1-f)^{2}$ where $\phi(s)=\mu(1-s)-1+f$. Since $\phi(1)=0$, and $\phi^{\prime}(s)<0$ for $s<1$, so $\phi(s) \geq 0$ for $s \leq 1$.

Example 6 Binomial distribution $B(n, p)$. In this case, $f=(1-p+s p)^{n}$ and we can similarly verify that the derivative of $(1-s) f^{\prime} /(1-f)$ is non-negative.

Proof of Proposition 1. The regular tree is a Galton-Watson tree with the degenerated distribution: $p_{d}=1$. In this case $f(s)=s^{d}, f^{\prime}(s)=d s^{d-1}$ and

$$
\frac{(1-s) f^{\prime}}{1-f}=d \frac{s^{d-1}-s^{d}}{1-s^{d}}
$$

is indeed increasing for $s \in(0,1)$. The condition of Theorem 3 is satisfied and Proposition 1 is proved.

There is a close relation between the speed of the simple random walk on a graph and the anchored expansion constant of the graph. It is shown in [6] that a positive anchored expansion constant implies that the speed is positive. We also know from [2] that the anchored expansion constant of the infinite cluster is positive if the 
anchored expansion constant of the original graph is positive and if the retaining probability of the Bernoulli bond percolation is close to 1. In light of the previous discussion we may ask the following question.

Question B: Is the anchored expansion constant of an infinite cluster of a transitive graph increasing in $p$ ? Is it continuous in $p$ ?

Although a negative answer would be very surprising, we even do not know the answer for a regular tree. In this case the infinite cluster is just a Galton-Watson tree and the anchored expansion constant is a constant a.s. for all Galton-Watson trees. It is positive and is less than $m-1$, where $m$ is the mean number of offsprings [2]. A moment of thought reveals that the inequality could be strict.

\section{Galton-Watson Trees}

Take a Galton-Watson tree with offspring distribution $\left\{p_{k}, k=0,1,2, \cdots\right\}$ and consider the Bernoulli bond percolation on the tree with retaining probability $p$. The open cluster containing the root $o$ is again a Galton-Watson tree with offspring distribution

$$
\bar{p}_{l}=\sum_{r=0}^{\infty} p_{l+r} p^{l}(1-p)^{r} C_{l+r}^{r}, \quad \text { for } l=0,1,2, \cdots .
$$

In order to have an infinite cluster, the retaining probability $p>1 / m_{1}$ where $m_{1}=$ $\sum_{k} k p_{k}$ is the mean number of offsprings.

We are now working with two Galton-Watson processes: $\left\{p_{k}\right\}$ and $\left\{\bar{p}_{k}\right\}$. The extinction probability is denoted by $q$ and $\rho$ respectively. The mean of the former is denoted by $m_{1}$. The generating function $f(s)=\sum_{k} p_{k} s^{k}$ is exclusively for the former.

Lemma $2.1 \rho$ is monotone and differentiable in $p$ for $p \in\left(1 / m_{1}, 1\right)$. Let $\lambda=$ $1-p+p \rho$. Then

$$
\frac{d \rho}{d p}=-\frac{(1-\rho) f^{\prime}(\lambda)}{1-p f^{\prime}(\lambda)}
$$

Proof. By definition $\rho$ satisfies the following equation.

$$
\rho=\sum_{k=0}^{\infty} \bar{p}_{k} \rho^{k}=\sum_{l=0}^{\infty} p_{l}(1-p+p \rho)^{l} .
$$


Equivalently,

$$
\frac{\lambda-1+p}{p}=\rho=f(\lambda)
$$

or

$$
p=\frac{1-\lambda}{1-f(\lambda)} .
$$

So $p$ is continuous and differentiable in $\lambda$. Except the degenerated case that $f(s)=$ $s, d p / d \lambda<0$ by the convexity of $f$. Therefore $\lambda$ as the inverse function is also continuous and differentiable in $p$. Take the derivative of the both sides of (6) with respect to $p$ we get,

$$
\frac{d \lambda}{d p}=-\frac{1-f(\lambda)}{1-p f^{\prime}(\lambda)} .
$$

This together with (5) implies (3). As a by-product, $1-p f^{\prime}(\lambda)>0$.

Remark: We know little of $\rho$. Even for a regular tree, we only have a good estimate of $\rho$.

$$
\rho(p) \leq \frac{1-p}{d^{2} p^{2}-p} \quad \text { for } p \in(1 / d, 1) .
$$

An exceptional case is the binary tree for which $\rho=(1-p)^{2} / p^{2}$ can be found explicitly, and is used in Example 2.

We assume that the open cluster $\mathcal{C}_{o}$ containing the root is infinite. As a GaltonWatson tree, $\mathcal{C}_{o}$ can be constructed as follows, see [4]. Begin with the root which is declared to be green. Add to the root a random number of edges according to probability distribution $P(Y=k)=\bar{p}_{k}\left(1-\rho^{k}\right) /(1-\rho)$. Declare the other end vertex of newly-added edge green with probability $1-\rho$ and red with probability $\rho$, independent of each other. If all the newly added vertices are red, discard the entire assignment and reassign green/red all over again. For each green vertex, repeat the same procedure. For each red vertex, attach to it independently a random number of red vertices according to the distribution $\hat{p}_{k}=\bar{p}_{k} \rho^{k-1}$ for $k \geq 0$. The infinite tree consisting of green vertices will be called the backbone and a connected component of red vertices is called a bush. The backbone is a Galton-Watson tree generated according to the distribution:

$$
\tilde{p}_{k}=\sum_{r=0}^{\infty} \bar{p}_{k+r} \rho^{r}(1-\rho)^{k-1} C_{k+r}^{r} \quad \text { for } k \geq 1 .
$$


Notice that

$$
\tilde{p}_{k}=\sum_{n=k}^{\infty} p_{n} C_{n}^{k} \lambda^{n-k} p^{k}(1-\rho)^{k-1} .
$$

Lemma 2.2 The speed of the simple random walk on the backbone is increasing in $p$ if $(1-s) f^{\prime}(s) /(1-f(s))$ is increasing in $s$. It is continuous for all $p \leq 1$ and differentiable for $p \in\left(1 / m_{1}, 1\right)$.

Proof. Let $S(p)$ be the speed of the simple random walk on the backbone. By formula (1),

$$
\begin{aligned}
S(p) & =\sum_{k=1}^{\infty} \tilde{p}_{k} \frac{k-1}{k+1}=1-2 \sum_{k} \tilde{p}_{k} \frac{1}{k+1} \\
& =1-2 \sum_{k=1} \frac{1}{k+1} \sum_{n=k}^{\infty} p_{n} C_{n}^{k} \lambda^{n-k} p^{k}(1-\rho)^{k-1} \\
& =1+\frac{2 \rho}{1-\rho}-\frac{2}{(1-\rho)^{2}} \sum_{l=0}^{\infty} \sum_{r=0}^{\infty} p_{l+r} p^{l}(1-p)^{r} C_{l+r}^{r} \frac{1-\rho^{l+1}}{l+1} \\
& =\frac{1+\rho}{1-\rho}-\frac{2}{(1-\rho)^{2} p} \sum_{n=0}^{\infty} \frac{p_{n}}{n+1} \sum_{l+r=n} \frac{(n+1) !}{r !(l+1) !} p^{l+1}(1-p)^{r}\left(1-\rho^{l+1}\right) \\
& =\frac{1+\rho}{1-\rho}-\frac{2}{(1-\rho)^{2} p} \sum_{n=0}^{\infty} \frac{p_{n}}{n+1}\left[1-(1-p+p \rho)^{n+1}\right] \\
& =\frac{1+\rho}{1-\rho}-\frac{2}{(1-\rho)^{2} p} \sum_{n=0}^{\infty} p_{n} \frac{1-\lambda^{n+1}}{n+1} .
\end{aligned}
$$

The continuity and differentiability now follow from Lemma 2.1. Take the derivative of $S(p)$ with respect to $p$, using (4) in the third equation.

$$
\begin{aligned}
S^{\prime}(p) & =\frac{2 \rho^{\prime}}{(1-\rho)^{2}}+\frac{2}{(1-\rho)^{2} p} \sum_{n=0}^{\infty} p_{n} \lambda^{n} \lambda^{\prime}-2 \frac{2 p \rho^{\prime}-(1-\rho)}{(1-\rho)^{3} p^{2}} \sum_{n=0}^{\infty} p_{n} \frac{1-\lambda^{n+1}}{n+1} \\
& =\frac{2}{(1-\rho)^{2}}\left[\rho^{\prime}+\frac{\rho}{p}\left(-1+\rho+p \rho^{\prime}\right)-\frac{2 p \rho^{\prime}-(1-\rho)}{(1-\rho) p^{2}} \sum_{n=0}^{\infty} p_{n} \frac{1-\lambda^{n+1}}{n+1}\right] . \\
& =\frac{2}{(1-\rho)^{2}}\left[-(1+\rho) \frac{(1-\rho) f^{\prime}}{1-p f^{\prime}}-\frac{\rho(1-\rho)}{p}+\frac{1+p f^{\prime}}{\left(1-p f^{\prime}\right) p^{2}} \sum_{n=0}^{\infty} p_{n} \frac{1-\lambda^{n+1}}{n+1}\right] \\
& =\frac{2 \Psi(p)}{(1-\rho)^{2}\left(1-p f^{\prime}\right) p^{2}}
\end{aligned}
$$

where

$$
\Psi(p)=-(1-\rho) p\left(\rho+p f^{\prime}\right)+\left(1+p f^{\prime}\right) \sum_{n} p_{n}\left(1-\lambda^{n+1}\right) /(n+1)
$$


Furthermore, $\Psi\left(1 / m_{1}\right)=0$ and

$$
\Psi^{\prime}(p)=\left[p(1-\rho)-\sum_{n} p_{n} \frac{1-\lambda^{n+1}}{n+1}\right]\left(-f^{\prime}-p f^{\prime \prime} \lambda^{\prime}\right) .
$$

It is enough to show $\Psi^{\prime}(p) \geq 0$ for $p \in\left(1 / m_{1}, 1\right)$. This can be done in two parts. First,

$$
p(1-\rho)-\sum_{n} p_{n} \frac{1-\lambda^{n+1}}{n+1} \geq 0,
$$

since its value at $p=1 / m_{1}$ is 0 and its derivative with respect to $p$ is $(1-\rho)^{2} /(1-$ $\left.p f^{\prime}(\lambda)\right) \geq 0$. Second,

$$
-f^{\prime}-p f^{\prime \prime} \lambda^{\prime}=-\frac{d}{d p}\left(p f^{\prime}(\lambda)\right)=\frac{d}{d \lambda}\left(\frac{(1-\lambda) f^{\prime}(\lambda)}{1-f(\lambda)}\right)\left(-\frac{d \lambda}{d p}\right) \geq 0
$$

by the assumption that $(1-s) f^{\prime}(s) /(1-f(s))$ is increasing in $s$.

Proof of Theorem 3. The speed of the simple random walk on the infinite cluster $\mathcal{C}_{o}$ can be calculated directly by applying (2). In our notation,

$$
\begin{aligned}
\text { Speed } & =\sum_{k=0}^{\infty} \bar{p}_{k} \frac{k-1}{k+1} \frac{1-\rho^{k+1}}{1-\rho^{2}} \\
& =1-\frac{2}{\left(1-\rho^{2}\right) p} \sum_{n=0}^{\infty} p_{n} \frac{1-\lambda^{n+1}}{n+1} \\
& =\frac{1-\rho}{1+\rho} S(p),
\end{aligned}
$$

where $S(p)$ is defined in Lemma 2.2. It is monotone, continuous and differentiable by Lemmas 2.1 and 2.2 .

Acknowledgement: We would like to thank Yueyun $\mathrm{Hu}$ for stimulating discussions on Question A. This paper is completed while the first author is visiting MSRI, its generous support is gratefully acknowledged.

\section{References}

[1] Benjamini, I., Lyons, R. Schramm, O. (1999) Percolation Perturbations in Potential Theory and Random Walks, in Random walks and Discrete Potential Theory. Cambridge Univ. Press, 56-84.

[2] D. Chen Y. Peres, (2004) Anchored expansion, percolation and speed, (with an appendix by Gaber Pete), Ann. of Probab., 32, 2978-2995. 
[3] Grimmett, G.R., Kesten, H. Zhang, Y. (1993) Random walk on the infinite cluster of the percolation model, Probab. Th. Rel. Fields, 96, 33-44.

[4] Lyons, R. (1992) Random walks, capacity, and percolation on trees, Ann. Probab., 20, 2043-2088.

[5] Lyons, R., Pemantle, R. Peres, Y. (1995) Ergodic theory on Galton-Watson trees: speed of random walk and dimension of harmonic measure, Ergodic Theory Dynamical Systems, 15, 593-619.

[6] Virág, B. (2000) Anchored expansion and random walk, Geom. Func. Anal. 10, 15881605 .

LMAM, School of Mathematical Sciences, Peking University, Beijing 100871, China

E-mail: dayue@math.pku.edu.cn, zhangfxi@math.pku.edu.cn 